\title{
Análisis de la situación bucal chilena.
}

Estimado Sr. Editor:

La siguiente carta tiene como finalidad expresar las deficiencias sobre los indicadores de caries utilizados para evaluar la salud oral de la población chilena y las cifras expresadas a la Organización Mundial de la Salud (OMS). A nivel mundial, la enfermedad de caries no tratada en dientes permanentes es la enfermedad crónica más prevalente y en dientes temporales es la décima ${ }^{1}$.

El indicador de caries más utilizado, a nivel mundial, es el índice ceod, en dientes temporales, y COPD, en dientes permanentes, el cual es una variable cuantitativa continua que indica la historia de caries y es capaz de detectar las lesiones cavitadas con dentina expuesta, sin detectar aquellas incipientes ni su actividad, con un sesgo sobre la causa de ausencia dentaria, elementos tan importantes para realizar un correcto diagnóstico. Es de fácil aplicación y análisis en estudios epidemiológicos, siendo el más estudiado y utilizado por la OMS.

La principal edad utilizada para medir y comprar la salud oral de la población a nivel mundial es a los 12 años, en donde los dientes permanentes han erupcionado recientemente, sin estar expuestos al medio oral un tiempo suficiente para desarrollar lesiones cavitadas.

El último informe realizado por el MINSAL data de diciembre del 2010, no representando la salud oral de la población actual. Además, es un estudio basado en el índice COPD solo detectando lesiones de caries cavitadas sin expresar la verdadera realidad. Por ejemplo, la prevalencia de lesiones de caries a los 6 años es de un 70,4\% con un ceod de 3,7 y un COPD de $0,15^{2}$. Al utilizar un índice con una capacidad de detectar lesiones de caries incipientes, ICDAS, por ejemplo, la prevalencia sería aún mayor mostrando una menor cantidad de dientes sanos que con el índice ceod/COPD con diferencias estadísticamente significativas ${ }^{3}$, demostrando la importancia del indicador seleccionado y el impacto que puede tener en los resultados que se adquieren. ICDAS es una variable cualitativa de uno o dos dígitos, el primer dígito indica la historia del diente (tipo de restauración o ausencia de diente discriminando la causa), el segundo dígito indica presencia/ausencia y severidad de lesión de caries coronal o radicular. Entrega una mayor sensibilidad y la actividad de la lesión, aunque es operador dependiente, requiere de aire comprimido y profilaxis previa para su análisis, y la combinación de variables entrega múltiples combinaciones, factores que dificultan su aplicación en estudios poblacionales y análisis estadístico.

Si consideramos que el esmalte de un diente temporal es más poroso, delgado y presenta un menor contenido mineral respecto a los dientes permanentes, evidenciamos características que determina un avance de mayor magnitud y velocidad de lesiones incipientes hacia cavitadas, siendo fundamental detectarlas a tiempo adquiriendo una visión preventiva y no reactiva, reconociendo toda lesión de caries y no solo las cavitadas.

A los 12 años, los dientes permanentes presentan una escasa exposición a los factores 
de riesgo cariogénicos; además, se debe considerar que son más resistentes que los dientes temporales frente a estos ataques. Por esta razón, es fundamental utilizar indicadores capaces de detectar lesiones incipientes debido a que, a esta edad, es más probable detectarlas en comparación a aquellas que se encuentran cavitadas. En base a este análisis, el último informe de salud bucal del país subestima la enfermedad de caries, lo que podría explicar el excesivo aumento del índice COPD en tres años, al incrementar de un 1,9 a los 12 años hasta un 3,0 a los 15 años ${ }^{2}$, cifras que aumentan dramáticamente en la población adulta y que pueden ser resultado de no detectar lesiones incipientes que posteriormente se cavitan, situación que requiere de nuevos estudios.

Esto puede hacernos cuestionar, ¿por qué la población chilena presenta un alto deterioro de la salud oral? Es probable que la respuesta esté en relación con la visión que expresan estos estudios, lo que influye en la preferencia de tratamientos invasivos en desmedro de los preventivos y estrategias de promoción de salud, inclusivas a toda la población. Esto es crucial en la población infantil debido a que estos tipos de tratamientos conllevan el uso de anestésicos, exodoncias o instrumental rotatorio con una alta susceptibilidad por generar experiencias dolorosas, traumáticas e incluso temor, marcando su desarrollo, pudiendo generar fobias a la atención dental que pueden afectar su compromiso con su salud oral durante su adultez, limitándose a buscar atención odontológica solo cuando sus patologías se encuentran avanzadas y las opciones terapéuticas son limitadas ${ }^{1}$.

Esta evidencia muestra que para contribuir a mejorar la salud oral de la población se debe comenzar por detectar la prevalencia y el impacto de esta patología en la población de forma fidedigna y con indicadores que permitan una mayor precisión, pero sin perder la facilidad y capacidad de emplearse en estudios epidemiológicos, situación que cumple el índice ceod/COPD ${ }^{1}$, y que podría adaptarse con el uso de ICDAS fusionado, aunque perdiendo sensibilidad por lo que se requiere formular un indicador que integre las fortalezas de ceod/COPD e ICDAS. De esta forma, es posible implementar políticas de salud pública para mejorar los índices de caries, tratando la enfermedad como tal y no la manifestación clínica con lesiones avanzadas. Es importante poner énfasis en la población infantil buscando mejorar sus experiencias, junto a buscar disminuir las paupérrimas tasas de morbilidad en la población adulta, las que, incluso, alcanzan un $100 \%$ de prevalencia de lesiones de caries ${ }^{1,4}$. Como define la OMS individuo sano es aquel que tiene un "bienestar físico, mental y social, y no solamente la ausencia de afección o enfermedades", en donde la enfermedad de caries perturba a los afectados provocando vergüenza, retraimiento social y afecciones estéticas por la pérdida de tejido dentario, ya sea durante la patología activa o como una consecuencia de esta; incluso puede provocar alteraciones físicas como dolor y procesos infecciosos, siendo una patología que hay que considerar fervientemente al afectar la salud integral de nuestros pacientes, siendo necesario la realización de estudios nacionales actualizados, en diversos grupos etarios y con indicadores que incluyan lesiones incipientes.

Marcial Guiñez Coelho

DDS, GamaSmile, Santiago, Chile. 


\section{REFERECIAS}

[1] Guiñez M, Letelier G. Especificidad y sensibilidad de sistema ICDAS ${ }^{\text {TM }}$ versus índice COPD en la detección de caries. Int. J. Odontostomat. 2020; 14(1):12-8. DOI: $10.4067 / S 0718-$ $381 \times 2020000100012$.

[2] MINSAL. Análisis de situación salud bucal en Chile. [Internet]. MINSAL. 2010 [Consultado el 25 de septiembre 2020].

minsal.cl/sites/default/files/files/An\%c3 \%a1lisis\%20de\%20Situaci\%c3\%b3n\% 20Salud\%20Bucal\%20final\%20pdf.pdf

[3] Ferraz EG, Silva LR, Sarmento VA, Campos EJ, Oliveira TFL, et al. Comparison of two visual methods for detecting caries among obese and nonobese children. Acta odontol scand. 2016; 74(5): 405-10. DOI: 10.3109/00016357.2016.1169555

[4] Mariño RJ, Cueto A, Badenier O, Acevedo R, Moya R. Oral health status and inequalities among ambulant older adults living in central Chile. Community Dent Health. 2011; 28: 1438. DOI: 10.1922/CDH_2567Mariño06.

[5] OMS. ¿Cómo define salud la OMS?. Preguntas frecuentes. [Internet]. OMS. 2020 [Consultado el 25 de septiembre 2020]. who.int/es/about/who-weare/frequently-asked-questions

\section{CÓMO CITAR ESTE ARTÍCULO}

Guiñez M. Análisis de la situación bucal chilena. Appli. Sci. Dent. 2020; 1(3):1-3. DOI: 10.22370/asd.2020.1.3.2441 